\section{Paper Chromatography of Some Sulfinic Acids and Sulfonylchlorides}

L. GRINGRAS and G. SJÖTEDT

\section{The Laboratory of AB Ceaverken, Strängnäs, Sweden}

Feigl $^{1}$ used the orange-red insoluble iron(III) salt of benzenesulfinic acid as spot test. Gilman and Abbot ${ }^{2}$ characterized $p$-toluenesulfinic acid by the melting point of thalliumdichloride- $p$-toluenesulfinate.

It has now been found that alkyl and arylsulfinic acids (or their salts) react with zinc chloride stabilized tetrazo-0-dianisidine

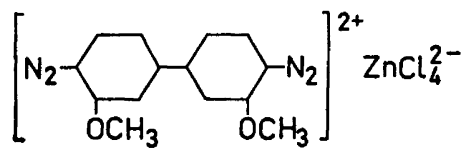

(Echtblau B Salz, E. Merck) to give a bright canary yellow dye, insoluble in water but soluble in benzene. Thus a drop of a solution of a sulfinic acid or a sulfinate $a b$ sorbed on filter paper and dried, appears as a yellow spot when treated with a freshly prepared $0.5 \%$ aqueous solution of the reagent. Thiosulfate, sulfite and thiocya. nate react in the same way to give yellow dyes, but these dyes are easily soluble in cold water and can be completely removed from the spot by washing it. Aromatic sulfonic acids, sulfones, mercaptans, phenols and disulfides do not interfere.

It has also been found that solutions of sulfinic acids or their salts can be examined by ascending paper chromatography, using a mixture of equal volumes of $n$-butanol, $n$-propanol and water, as the solvent.

Essentially the same technique has been used for the analysis of alkyl and arylsulfonylchlorides except that the marked spot on the paper was pre-treated with an alkaline sodium sulfite solution before applying the sulfonylchloride. In this way the sulfonylchloride was converted to the sulfinic salt directly on the paper, before starting the chromatographic run.

Separation of mixtures of sulfinic acids or sulfonylchlorides was possible providing the $R_{F}$-values differed enough.

The following substances were investigated:

Acta Chem. Scand. 15 (1961) No. 2
Sulfinic acids:

Benzenesulfinic acid and salts (Hoechst) $p$-Toluenesulfinic acid (Bofors, Sweden) 2,6-Dichloro- $p$-toluenesulfinic acid (prepared according to Ref. ${ }^{3}$ )

Ethansulfinic acid, sodium salt (Hoechst) 2-Naphtalenesulfinic acid ${ }^{3}$

Sulfonylchlorides:

$p$-Toluenesulfonylchloride (Bofors, Sweden)

2,6-Dichloro- $p$-toluenesulfonylchloride (Bofors)

2-Naphthalenesulfonylchloride (Merck, Germay)

Methansulfonylchloride (Fluka, Switzerland)

Whatman No. 1 filter paper for chroma tography, $24 \times 40 \mathrm{~cm}$ in size was used. The chromatograms were developed in a suitably sized glass tank equipped with stainless steel racks from which the paper was suspended so es to dip into the solvent, which was placed at the bottom of the tank. The chromatograms were developed for $16 \mathrm{~h}$ at $20^{\circ} \mathrm{C}$. The solvent consisted of chromatographically pure $n$-butanol, $n$ propanol and water $(1: 1: 1)$.

Concentration standards, $5 \times 10^{-8}$ and $5 \times 10^{-2} \mathrm{M}$, were prepared from sulfinic acid salts in distilled water.

The sulfonylchlorides were dissolved in dioxan, ethanol or benzene. Liquid sulfonylchlorides were used directly, without dilution.

Detection of sulfinic acids. The standard solutions were applied from a glass capillary on a starting line $3 \mathrm{~cm}$ from the bottom edge of the paper sheet and $2 \mathrm{~cm}$ intervals between spots, by touching the end of the capillary to the paper and withdrawing it when the spot had attained the required size. The spots could be made visible after developing and drying the chromatogram, by spraying it with a dilute solution of potassium permanganate, when the sulfinic acid appeared as a white spot on the purple background. After some time, however, the purple background faded out. A spray

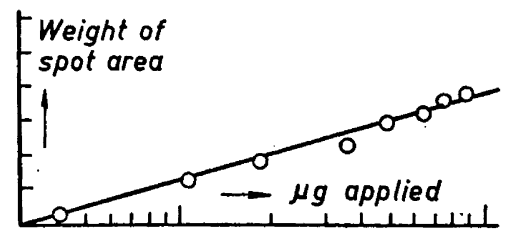

Fig. 1. Quantitative estimation of the amount of $p$-toluenesulfinic acid as a function of spot area. 
Table 1.

\begin{tabular}{|c|c|c|c|}
\hline Substance & $R_{F}$ & Substance & $R_{F}$ \\
\hline Benzenesulfinic acid & 0.58 & 2,6-Dichloro- $p$-toluenesulfinic acid & $0.81^{*}$ \\
\hline$p$-Toluenesulfinic acid & 0.65 & Ethansulfinic acid & 0.40 \\
\hline 2-Naphtalenesulfinic acid & 0.73 & Methansulfinic acid & $0.25^{* *}$ \\
\hline
\end{tabular}

* Practical grade salt gave a faint second spot (0.79).

** From reduced methansulfonyl chloride.

consisting of a solution of dinitrophenylindole gave white spots on a blue background. Neither of these reagents are specific for the sulfinic group. The insoluble yellow dye formed by spraying with a $0.25-0.5 \%$ solution of the stabilized tetrazotized 0 dianisidine, seems to be very specific. Darkening of the white background on storage can be prevented by washing the chromatogram with cold distilled water.

Detection of sulfonylchlorides. First a spot of a $20 \%$ sodium hydroxide solution saturated with sodium sulfite was applied to a marked position on the starting line. This was dried and a spot of the solution of sulfonylchloride (or the mixture of sulfonylchlorides) was superimposed on it. By holding the paper for a short time over a dish containing hot or boiling water, or by conditioning it in a humidity cabinet for an hour, the sulfonylchloride was reduced to the parent sulfinic salt. If several spots were applied they were spaced at intervals of about $5 \mathrm{~cm}$. After developing and spraying the chromatogram, the characteristic spots of the sulfinic group afpeared. The sulfite spot, which had a rather large area due to the high sulfite concentration, disappeared when the chromatogram was washed.

The $R_{F}$-values obtained are reported in Table 1 .

Weighed amounts of a standard solution of sodium $p$-toluenesulfinate were applied from a capillary pipet to the chromatographic paper. If recessary the loading was increased by multiple applications, each spot being dried before repeating the application. In this way series of spots corresponding to from 0.5 to $80 \mu \mathrm{g}$ of the salt was obtained. The minimum quantity detectable after developing, drying and spraying the chromatogram as previously described was found to be $4 \mu \mathrm{g}$ which is equal to $2.4 \times 10^{-8}$ mole of sodium $p$ toluenesulfinate.

A quantitative estimation was carried out using the technique devised by Fisher et al. The spots from a concentration series were circumscribed with a black pencil and copied on photographic document reflex paper or on transparent paper of uniform quality. The spots thus obtained are carefully cut out and weighed. By plotting on semi-logarithmic paper the weights of these "cut-outs" against the amounts of sulfinic salt applied on each spot, a straight calibration curve was obtained (Fig. 1), from which the content of an unknown sample could be determined.

The sulfonylchlorides could be examined in the same way provided the reduction was complete. Amounts as small as $100 \mu \mathrm{g}$ could be detected without difficulty.

The 8uthors wish to thank the Directors of $\mathrm{AB}$ Ceaverken for permission to publish this paper.

1. Feigl, F. Laboratory Manual of Spot Tests. New York 1943, p. 181-3; Feigl, F. Quantitative Analysis of Spot Tests. New York 1947 , p. 366-7.

2. Gilman, H. and Abbot, R. K. J. Am. Chem. Soc. 71 (1949) 659.

3. Organic Syntheses, Coll. Vol. I (1947) 492.

4. Block, R. J., Durrum, E. L. and Zweig, G. A Manual of Paper Chromatography and Electrophoresis. New York 1955, p. 56.

Received January 28, 1961. 